\title{
Both Eyes
}

National Cancer Institute

\section{Source}

National Cancer Institute. Both Eyes. NCI Thesaurus. Code C119335.

Of or pertaining to both the left and right eyes, 